\title{
Carbonaceous molecules in the oxygen-rich circumstellar environment of binary post-AGB stars ${ }^{\star}$
}

\section{$\mathrm{C}_{60}$ fullerenes and polycyclic aromatic hydrocarbons}

\author{
C. Gielen ${ }^{1,2, \star \star}$, J. Cami ${ }^{3,4}$, J. Bouwman ${ }^{2}$, E. Peeters ${ }^{3,4}$, and M. Min ${ }^{5}$ \\ 1 Instituut voor Sterrenkunde, Katholieke Universiteit Leuven, Celestijnenlaan 200D, 3001 Leuven, Belgium \\ e-mail: clio.gielen@ster.kuleuven.be \\ 2 Max Planck Institut für Astronomie, Königstuhl 17, 69117 Heidelberg, Germany \\ 3 Department of Physics and Astronomy, University of Western Ontario, London, Ontario N6A 3K7, Canada \\ 4 SETI Institute, 189 Bernardo Ave, Suite 100, Mountain View, CA 94043, USA \\ 5 Astronomical Institute, University of Utrecht, PO Box 80000, 3508 TA Utrecht, The Netherlands \\ Received 27 August 2011 / Accepted 19 October 2011
}

\section{ABSTRACT}

\begin{abstract}
Context. The circumstellar environment of evolved stars is generally rich in molecular gas and dust. Typically, the entire environment is either oxygen-rich or carbon-rich, depending on the evolution of the central star.

Aims. In this paper we discuss three evolved disc sources with evidence of atypical emission lines in their infrared spectra. The stars were taken from a larger sample of post-AGB binaries for which we have Spitzer infrared spectra, characterised by the presence of a stable oxygen-rich circumbinary disc. Our previous studies have shown that the infrared spectra of post-AGB disc sources are dominated by silicate dust emission, often with an extremely high crystallinity fraction. However, the three sources described here are selected because they show a peculiar molecular chemistry.

Methods. Using Spitzer infrared spectroscopy, we study in detail the peculiar mineralogy of the three sample stars. Using the observed emission features, we identify the different observed dust, molecular and gas species.

Results. The infrared spectra show emission features due to various oxygen-rich dust components, as well as $\mathrm{CO}_{2}$ gas. $\mathrm{All}$ three sources show the strong infrared bands generally ascribed to polycyclic aromatic hydrocarbons. Furthermore, two sample sources show $\mathrm{C}_{60}$ fullerene bands.

Conclusions. Even though the majority of post-AGB disc sources are dominated by silicate dust in their circumstellar environment, we do find evidence that, for some sources at least, additional processing must occur to explain the presence of large carbonaceous molecules. There is evidence that some of these sources are still oxygen-rich, which makes the detection of these molecules even more surprising.
\end{abstract}

Key words. stars: AGB and post-AGB - binaries: general - circumstellar matter - stars: abundances

\section{Introduction}

The chemistry of the circumstellar environment (CE) of evolved stars can typically be described as either oxygen-rich or carbonrich, and reflects the evolution of the central object along the Hertzsprung-Russell diagram. Stars on the asymptotic giant branch (AGB) start as oxygen-rich stars, but their carbon abundance rises during the evolution on the AGB, since the carbon produced in the core is dredged-up to the stellar photosphere. If this dredge-up process repeats enough times, carbon becomes more abundant than oxygen: the star has become a carbon star. The main parameters determining the mass of carbon dredged up are the initial mass of the central star and its metallicity (see Herwig 2005, for a detailed description of AGB evolution).

Depending on the $\mathrm{C} / \mathrm{O}$ ratio of the central star, its $\mathrm{CE}$ will be either oxygen-rich or carbon-rich. The less abundant species will be locked in carbon monoxide (CO), which is an extremely

\footnotetext{
* This research has made use of the SIMBAD database, operated at CDS, Strasbourg, France. Based on observations made with the Spitzer Space Telescope (program id 3274, 50092), which is operated by the Jet Propulsion Laboratory, California Institute of Technology under a contract with NASA.

$\star \star$ Postdoctoral Fellow of the Fund for Scientific Research, Flanders.
}

stable molecule and one of the first to be formed. If the central star is oxygen-rich, the CE is typically characterised by gas and dust features such as from $\mathrm{OH}, \mathrm{CO}_{2}, \mathrm{H}_{2} \mathrm{O}$, oxides, and silicates (e.g. Justtanont et al. 1996; Waters et al. 1996; Molster et al. 2002a; Gielen et al. 2011). For some oxygen-rich sources, the presence of carbon-rich molecules can be explained by nonequilibrium effects, such as shocks (Nejad \& Millar 1988; Duari et al. 1999; Cherchneff 2006). For example, S-type stars, which are undergoing the transition from oxygen-rich to carbon-rich and thus have $\mathrm{C} / \mathrm{O} \sim 1$, do not yet form carbon-rich dust. However, due to non-equilibrium chemistry effects carbon-rich molecules, such as PAHs, $\mathrm{HCN}$, and $\mathrm{C}_{2} \mathrm{H}_{2}$ can be seen (Hony et al. 2009; Smolders et al. 2010). In the carbon-rich case, the excess carbon is used to form, for example, $\mathrm{CN}, \mathrm{C}_{2}, \mathrm{C}_{2} \mathrm{H}_{2}, \mathrm{CH}_{4}$, polycyclic aromatic hydrocarbons (PAHs), and SiC. (e.g. Bakker et al. 1997; Speck et al. 1997; Peeters et al. 2002; Hony et al. 2003; Matsuura et al. 2006; Volk et al. 2011). Recently, even more complex carbon molecules, namely $\mathrm{C}_{60}$ and $\mathrm{C}_{70}$ fullerenes, were detected in the CE of evolved stars (Cami et al. 2010; García-Hernández et al. 2010, 2011; Zhang \& Kwok 2011).

In some cases, a strong mixed chemistry is seen in the CE of evolved objects (Szczerba et al. 2007). One of these is a particular class of AGB stars, the silicate carbon stars, where 
the infrared spectra show features of both C-rich species and O-rich silicates (Yamamura et al. 2000; Molster et al. 2001; García-Hernández et al. 2006). Also some post-AGB stars and proto-planetary nebulae (pPNe) show features of both PAHs and silicates in their spectra (Beintema et al. 1996; Waters et al. 1998; Matsuura et al. 2004; Cerrigone et al. 2009; GuzmanRamirez et al. 2011). For all these objects the dual chemistry is usually a result of the evolution of the central star. The O-rich matter was expelled when the star was still oxygenrich, followed by a transition from $\mathrm{C} / \mathrm{O}<1$ to $\mathrm{C} / \mathrm{O}>1$ in the stellar photosphere. Subsequent outflow of material will then be carbon-rich. This effect is even more pronounced if the O-rich material is stored in a stable disc around the central star, as is the case for the silicate carbon stars and several of the mixed-chemistry pPNe (Waters et al. 1998; Deroo et al. 2007; Cerrigone et al. 2009).

In this paper we look in more detail to a sample of three postAGB disc sources. These sources are part of the larger sample of 57 post-AGB disc sources with Spitzer infrared spectra, as discussed in Gielen et al. (2011). In Gielen et al. (2011) we find that the circumstellar environment of these post-AGB disc sources is dominated by emission of silicate dust. However, four sources do show features attributed to carbonaceous molecules, in the form of PAHs and $\mathrm{C}_{60}$ fullerenes, besides the silicate emission. The carbonaceous content of one of these sources has already been discussed in Gielen et al. (2009). In this paper we focus on the remaining three stars.

Emission of PAHs is already observed in several post-AGB sources (e.g. Beintema et al. 1996; Cami \& Yamamura 2001; Peeters et al. 2002; Molster et al. 2002b; Matsuura et al. 2004; Gielen et al. 2009), where most of the central stars appear carbon-rich. There is evidence that the central stars from our sample are oxygen-rich, which would make the detection of carbonaceous molecules even more surprising. Moreover, this is the first time fullerenes are detected in O-rich post-AGB sources.

The outline of the paper is as follows: in Sect. 2 we present the programme stars and observation details. All sample stars show features due to O-rich dust species, which are discussed in Sect. 3. O-rich $\mathrm{CO}_{2}$ gas emission is also seen in a few sources, and discussed in Sect. 4. Clear emission of PAHs can be seen in the $6-12 \mu \mathrm{m}$ region of the observed infrared spectra, as can be seen in Sect. 5. In Sect. 6 we report on the detection of the $C_{60}$ fullerenes and derive some basic parameters such as the excitation temperature and mass. We end with discussions and conclusions in Sects. 7 and 8.

\section{Programme stars and observations}

The binary post-AGB stars discussed here are those sources from the larger Spitzer sample of post-AGB disc candidates (Gielen et al. 2011), showing features of carbonaceous molecules besides the dominant silicate emission seen in these stars. The sample of disc candidates was selected on the basis of their infrared colours, as discussed in De Ruyter et al. (2006) and Gielen et al. (2011) These binary post-AGB sources are characterised by the presence of a stable O-rich dust disc, with strong features of amorphous and crystalline silicates (Gielen et al. 2008, 2011). These post-AGB disc sources typically have an O-rich stellar photosphere, with no evidence for strong third dredge-up on the $\mathrm{AGB}$, as determined, for example, by the very low $\mathrm{C} / \mathrm{O}$ ratio $(\ll 1)$, low ${ }^{12} \mathrm{C} /{ }^{13} \mathrm{C}$ ratio, and the lack of s-process enhancement (e.g. Gonzalez et al. 1997; Van Winckel 2003; Maas et al. 2005; Reyniers \& Van Winckel 2007; Gielen et al. 2009). This is surprising since several stars have initial masses or luminosities

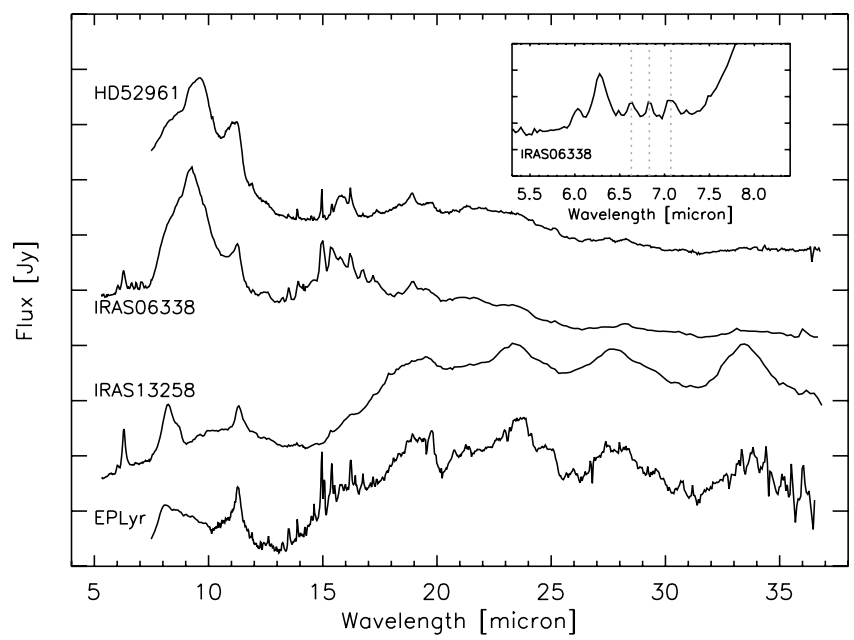

Fig. 1. The Spitzer-IRS high- and low-resolution spectra of our three sample stars IRAS 13258, IRAS 06338 and HD 52961, together with the spectrum of EP Lyr, which shows similar emission features. The spectra are scaled and offset for comparison. The inset plot shows the $7 \mu \mathrm{m}$ region of IRAS 06338 and IRAS 13258. The dotted gray lines mark the observed features at $6.63,6.83$ and $7.07 \mu \mathrm{m}$, probably due to small PAH, aliphatic hydrocarbons, and/or hydrogenated amorphous carbon emission.

high enough to evolve to a carbon star (Gielen et al. 2009, 2011). Apparently, the AGB evolution of these stars was shortcut, probably under the influence of strong binary interaction.

All stars were observed with the Spitzer-IRS spectrograph, in high- or low-resolution modes (resolution of $\sim 600$ and $\sim 160$ respectively Werner et al. 2004a; Houck et al. 2004). Exposure times were chosen to achieve a $\mathrm{S} / \mathrm{N}$ of $\sim 400$, and resulted in values of $2200 \mathrm{~s}, 1750 \mathrm{~s}$, and $300 \mathrm{~s}$ for IRAS 13258, IRAS 06338, and HD 52961, respectively. The spectra were extracted from the droop and RSC data products, processed through the S18.0 version of the SSC (Spitzer Science Centre) data pipeline. For the spectral extraction we used data reduction packages, developed for the $\mathrm{c} 2 \mathrm{~d}$ (Core to discs) and feps (Formation and evolution of planetary systems) key projects. For a detailed description of these reduction packages, we refer to Lahuis et al. (2006) and Hines et al. (2005). The resulting infrared spectra for our sample stars can be seen in Fig. 1. As shown in Gielen et al. (2011), the infrared spectra of these three stars show emission features which can not be explained by the typical silicate dust species, as is the case for the rest of the larger sample post-AGB disc sources. Details for the programme stars can be found in Table 1.

For IRAS $06338+5333$ (or HD 46703, IRAS 06338 hereafter) and HD 52961 the binarity is confirmed, and orbital parameters are given in Table 1 . These two stars also show the depletion pattern of condesable elements in the stellar photosphere (Waelkens et al. 1991b; Hrivnak et al. 2008), commonly seen in post-AGB disc sources (e.g. Giridhar et al. 2005; Maas et al. 2005). This abundance pattern, characterised by a lack of refractory elements, is the result of gas-dust separation in the disc, followed by a re-accretion of the cleaned gas component (Waters et al. 1992). HD 52961 is an extreme example of this process, having a $[\mathrm{Fe} / \mathrm{H}]$ ratio of -4.8 and $[\mathrm{Zn} / \mathrm{Fe}]=+3.1$. Chemical studies have shown that the stars are oxygen-rich, with C/O < 1 (Luck \& Bond 1984; Bond \& Luck 1987; Waelkens et al. 1991b; Van Winckel et al. 1992; Bakker et al. 1997; Hrivnak et al. 2008). Unfortunately, no C/O ratio has been determined for IRAS 13258-8103 (IRAS 13258 hereafter). Optical spectra obtained so far cannot give conclusive evidence for either a carbon- or oxygen-rich nature of the stellar photosphere 
Table 1. Name, equatorial coordinates $\alpha$ and $\delta(\mathrm{J} 2000)$, spectral type, effective temperature $T_{\text {eff }}$, surface gravity $\log g$ and metallicity [Fe/H] of our sample stars.

\begin{tabular}{|c|c|c|c|c|c|c|c|}
\hline Name & $\begin{array}{r}\alpha(\mathrm{J} 2000) \\
(\mathrm{h} \mathrm{m} \mathrm{s})\end{array}$ & $\begin{array}{r}\delta(\mathrm{J} 2000) \\
\left({ }^{\circ},{ }^{\prime \prime}\right)\end{array}$ & $\begin{array}{l}T_{\text {eff }} \\
(\mathrm{K})\end{array}$ & $\begin{array}{l}\log g \\
(\mathrm{cgs})\end{array}$ & {$[\mathrm{Fe} / \mathrm{H}]$} & $\begin{array}{r}P_{\text {orbit }} \\
\text { (days) }\end{array}$ & $\overline{\mathrm{C} / \mathrm{O}}$ \\
\hline IRAS $06338+5333$ & 063752.4 & +533102 & 6250 & 1.0 & -1.6 & 600 & 0.7 \\
\hline IRAS $13258-8103$ & 133107.1 & -811830 & F4Ib-G0Ib & & & & \\
\hline HD 52961 & 070339.6 & +104613 & 6000 & 0.5 & -4.8 & 1310 & 0.7 \\
\hline
\end{tabular}

Notes. For the model parameters we refer to Waelkens et al. (1991b), De Ruyter et al. (2006), Hrivnak et al. (2008). The spectral type for IRAS 13258 was determined from optical spectroscopy by Thomas Lloyd Evans (priv. comm.). Also given is the orbital period (see references in De Ruyter et al. 2006; Gielen et al. 2007) and the C/O ratio (Luck \& Bond 1984; Bond \& Luck 1987; Waelkens et al. 1991 b; Van Winckel et al. 1992; Hrivnak et al. 2008).

(Thomas Lloyd Evans, priv. comm.). So far, all post-AGB disc sources where we have studied the stellar photosphere, show an O-rich chemistry (e.g. Gonzalez et al. 1997; Giridhar et al. 1998, 2000; Van Winckel 2003). So it is most likely that this star also follows this trend. If not, and the photosphere is C-rich in stead, this would be the first C-rich post-AGB disc source detected.

All three sample stars show the typical broad infrared excess, associated with the presence of a circumbinary disc (De Ruyter et al. 2006; Giridhar et al. 2005). This excess stars already at near-infrared wavelengths, and is very different from the excess related to an outflowing shell, where the excess starts and peaks at much longer wavelengths. However, HD 52961 and IRAS 06338 do not show the very high $L_{\mathrm{IR}} / L_{*}$ ratio, seen in other post-AGB disc sources (see Fig. 1 in Gielen et al. 2011). For HD $52961 L_{\mathrm{IR}} / L_{*}=12 \%$ and IRAS 06338 has a ratio of only $3 \%$, whereas the typical value for such sources is around 40-50\% (Gielen et al. 2008, 2011). Although for IRAS 13258 the lack of stellar parameters and value for the total reddening makes an exact determination of the $L_{\mathrm{IR}} / L_{*}$ ratio difficult, the SED shows that the infrared excess is significant $(>50 \%$, Gielen et al. 2011). Lloyd Evans (1997) deduced that for this star the disc is most likely seen very edge on, since the strong Na D emission lines in the optical spectrum show that the star is seen by reflection on the circumstellar matter, which points to a close to edge-on orientation of the disc.

IRAS 06338 and HD 52961 have strong similarities with another post-AGB disc source, namely EP Lyr. This stars also has an O-rich classification (Gonzalez et al. 1997), and shows strong class $\mathrm{C}$ PAH features and $\mathrm{CO}_{2}$ gas line emission (see Fig. 1), together with strong features due to crystalline silicates (Gielen et al. 2009). Furthermore, EP Lyr also has an atypically low $L_{\mathrm{IR}} / L_{*}$ ratio of $3 \%$.

\section{Silicate dust features}

In order to study the carbonaceous molecules visible in the spectra, we need to remove the underlying silicate dust contribution. Our analysis of the residual carbonaceous emission is then further discussed in Sects. 5 and 6.

\subsection{The $10 \mu \mathrm{m}$ region}

The spectrum of IRAS 06338 is dominated by a broad emission feature peaking at $9.2 \mu \mathrm{m}$ (see Fig. 2). The emission is clearly different from the typical $9.8 \mu \mathrm{m}$ feature seen in other post-AGB disc sources (see Fig. 6 in Gielen et al. 2011), where the feature is mainly due to emission of amorphous and crystalline olivine. Clearly, other dust species, which peak at shorter wavelengths, are needed to explain the observed emission. One possibility is silica, which has different polymorphs that all peak around $9 \mu \mathrm{m}$ (Sargent et al. 2009). To determine the different contributing dust species to this feature, we assume the emission to come from an optically thin region and linearly add dust absorption profiles, as
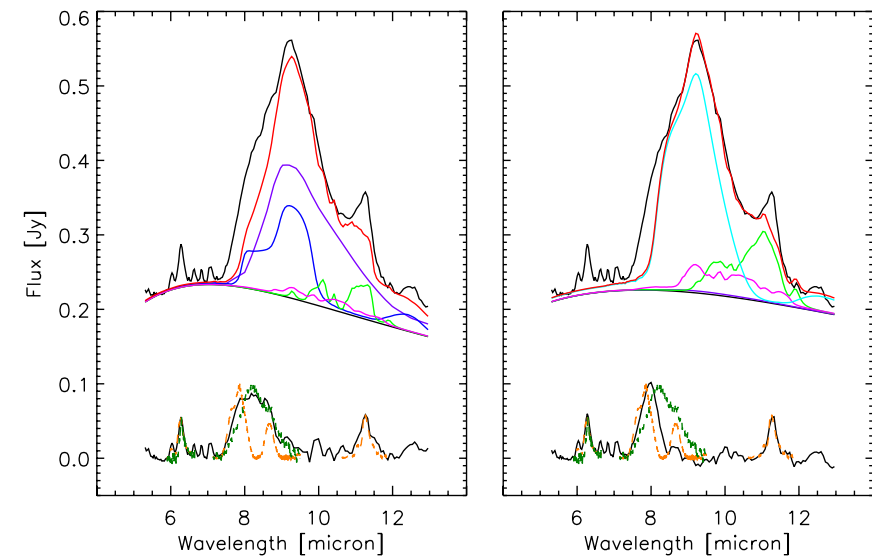

Fig. 2. Results of our modelling of the $10 \mu \mathrm{m}$ feature in IRAS 06338 , using silica (model A, left) and alumino-silicates (model B, right). The different dust contributions are: amorphous pyroxene (violet), amorphous silica (blue), enstatite (magenta), forsterite (light green) and $\mathrm{NaAlSi}_{4} \mathrm{O}_{10}$ (cyan). The combined spectrum is given in red. On the bottom we show the residuals of this model, and overplot class B (orange) and class C (dark green) PAH emission features as described by Peeters et al. (2002) and van Diedenhoven et al. (2004).

done in Gielen et al. (2008, 2011). The mass absorption coefficients for the dust species are calculated from refractory indices in gaussian random fields (GRF) dust approximation (Shkuratov $\&$ Grynko 2005). The refractive indices for the different dust species are taken from Servoin \& Pirou (1973); Dorschner et al. (1995); Henning \& Mutschke (1997), and Jaeger et al. (1998). Detailed results of the modelling can be found in Table A.1.

We can reproduce the feature using amorphous pyroxene, amorphous silica and forsterite (Fig. 2: Left). The residual emission at $6.04-6.28-8.23$ and $11.28 \mu \mathrm{m}$ can be explained by emission due to PAHs. Note that we do not find evidence for the presence of amorphous olivine, even though it is usually one of the most abundant species seen in the spectra of post-AGB disc sources (Gielen et al. 2008, 2011), and we do see features due to crystalline olivine at longer wavelengths (see Sect. 3.2). Some alumino-silicates also peak around $9 \mu \mathrm{m}$ (Mutschke et al. 1998), and a good fit can also be obtained using $\mathrm{NaAlSi}_{4} \mathrm{O}_{10}$, forsterite and enstatite (Fig. 2: Right). The residual spectrum is then very similar to the former case, but the $8.23 \mu \mathrm{m}$ residual feature is now blueshifted to $8.00 \mu \mathrm{m}$, with an additional feature at $8.66 \mu \mathrm{m}$. Again, the residual features can be explained by emission due to PAHs. In none of the models, adding amorphous olivine improved the fit. The models described here are most likely not the only solutions, since emission features of amorphous dust are often relatively interchangeable. However, all models show strong residual emission at $8 \mu \mathrm{m}$, but the exact shape remains unclear.

HD 52961 also shows a prominent feature in the $10 \mu \mathrm{m}$ region, peaking around $9.7 \mu \mathrm{m}$, pointing to the presence of 


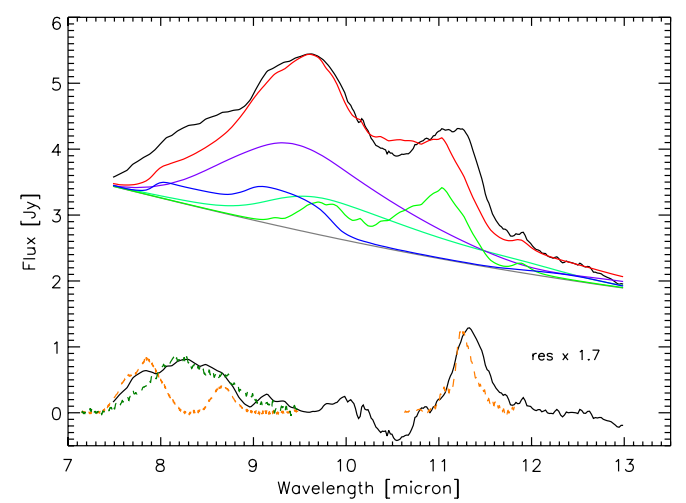

Fig. 3. Results of our modelling of the $10 \mu \mathrm{m}$ feature in HD 52961. The different dust contributions are: amorphous pyroxene (violet), amorphous olivine (turquoise), amorphous silica (blue) and forsterite (light green). The combined spectrum is given in red, with the continuum plotted in gray. On the bottom we show the scaled residuals of this model, and overplot an example of class B (orange) and class C (dark green) PAH emission features.

amorphous olivine and pyroxene. Again we see a clear shoulder at $8 \mu \mathrm{m}$ and a strong feature at $11.3 \mu \mathrm{m}$. This feature is much broader than what is observed for IRAS 06338 and is most likely a blend of forsterite and PAH emission. We are able to get a reasonable fit to the spectrum using amorphous olivine/pyroxene, amorphous silica and forsterite (see Fig. 3). However, it proves difficult to reproduce the strong $11.3 \mu \mathrm{m}$ without getting too much flux in the $10.5 \mu \mathrm{m}$ region. This could be due to the adopted synthetic spectrum for forsterite. The synthetic spectra of crystalline silicates are known to be very sensitive to the exact chemical composition, temperature, the grain shape and grain size (e.g. Koike et al. 2003, 2010). Similarly, also the very strong observed $16 \mu \mathrm{m}$ feature, generally associated with forsterite emission, proved difficult to reproduce with current forsterite synthetic spectra (Gielen et al. 2009, 2011). As for IRAS 06338, we again find residual emission at wavelengths corresponding to PAH emission features.

IRAS 13258 shows no strong silicate features in the $10 \mu \mathrm{m}$ region. A weak feature can be seen ranging from 8 to $13 \mu \mathrm{m}$. This could be due to weak emission of amorphous silicates. Since there is evidence that we see the disc close to edge on, this could be due to heavy reddening of the central star and the hot inner regions of the disc, where one would expect most of the $10 \mu \mathrm{m}$ emission to arise from.

\subsection{The $18-36 \mu m$ region}

For all three stars, the observed long-wavelength spectra show emission features that can be reproduced by emission of amorphous olivine/pyroxene, forsterite/enstatite and amorphous silica. In Fig. 4 we plot the observed long-wavelength spectra, together with model spectra consisting of the above mentioned dust species. Detailed results of the modelling can be found in Table A.1. Since the limited wavelength range makes it difficult to distinguish the underlying continuum from contribution of amorphous olivine/pyroxene, we only include amorphous silica, forsterite and enstatite in our modelling. $\mathrm{NaAlSi}_{4} \mathrm{O}_{10}$, which we used in the $10 \mu \mathrm{m}$ modelling of IRAS 06338, has a similar feature as silica at $21 \mu \mathrm{m}$, and so both dust species are interchangeable in this model. The features at $21 \mu \mathrm{m}$ of $\mathrm{NaAlSi}_{4} \mathrm{O}_{10}$ and $\mathrm{SiO}_{2}$ are relatively weak compared to the $10 \mu \mathrm{m}$ feature, especially at higher temperatures, so we do not expect to see a very strong contribution of these species at $21 \mu \mathrm{m}$.

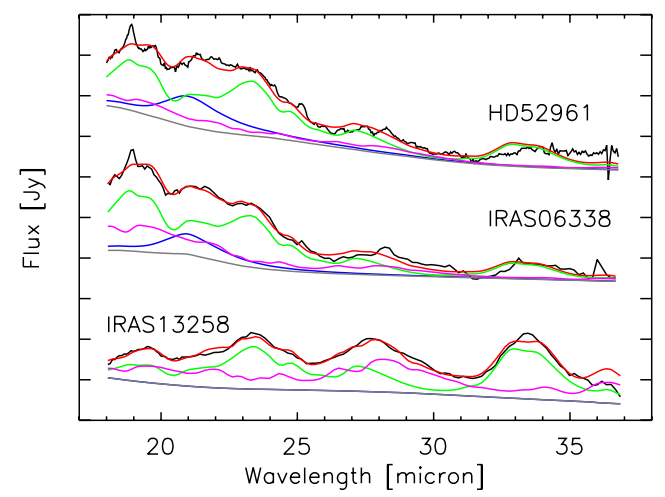

Fig. 4. The long-wavelength Spitzer spectrum of our sample stars, together with the model spectrum (red) consisting of amorphous silica (blue), forsterite (light green) and enstatite (magenta). The adopted continuum is given in gray. The spectra are scaled and offset for comparison.

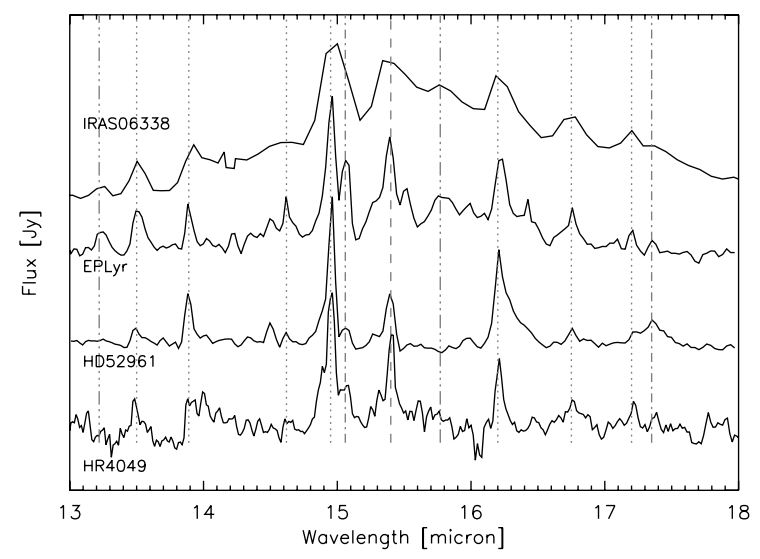

Fig. 5. The $\mathrm{CO}_{2}$ emission region of IRAS 06338, compared to other post-AGB sources showing $\mathrm{CO}_{2}$ gas emission (Cami \& Yamamura 2001; Gielen et al. 2009). Dotted lines show the location of ${ }^{12} \mathrm{C}^{16} \mathrm{O}_{2}$ features, dashed lines give ${ }^{13} \mathrm{C}^{16} \mathrm{O}_{2}$ and dot-dashed lines show ${ }^{16} \mathrm{O}^{12} \mathrm{C}^{18} \mathrm{O}$. Dot-dot-dashed lines mark unidentified features. The spectra are scaled and offset for comparison. For HD 52961, the underlying feature at $16 \mu \mathrm{m}$ was removed.

For IRAS 06338 and HD 52961, forsterite is clearly the dominant crystalline species, with a smaller contribution of enstatite and amorhous silica. In IRAS 13258, there is a significant contribution of enstatite, but there is no evidence for amorphous silica. For IRAS 13258 , the strong forsterite $33.6 \mu \mathrm{m}$ feature points to the presence of a large fraction of cool crystalline silicates in the disc. If we see the disc very edge on, we might see more of the cooler outlying material, explaining the dominance of the cool long-wavelength features in the spectrum.

\section{4. $\mathrm{CO}_{2}$}

In IRAS 06338 and HD 52961 we see strong narrow lines in the 13-17 $\mu$ m region, which can be identified with $\mathrm{CO}_{2}$ gas emission (Fig. 5).

For HD 52961, an underlying feature at $16 \mu \mathrm{m}$ is seen, probably (partly) coming from crystalline silicates, more specifically forsterite. The $\mathrm{CO}_{2}$ gas emission in IRAS 06338 is similar to the emission seen in HD 52961 and EP Lyr Gielen et al. (2009), and can also be identified with the $\mathrm{CO}_{2}$ isotopes ${ }^{12} \mathrm{C}^{16} \mathrm{O}_{2},{ }^{13} \mathrm{C}^{16} \mathrm{O}_{2}$ and ${ }^{16} \mathrm{O}^{12} \mathrm{C}^{18} \mathrm{O}$. Unfortunately, the low spectral resolution of IRAS 06338 does not allow to determine the exact isotope abundances, as was done for HD 52961.

The $\mathrm{CO}_{2}$ lines in IRAS 06388 seem to by lying on top a very broad $(\sim 4 \mu \mathrm{m})$ feature at $15 \mu \mathrm{m}$. Note that the $15 \mu \mathrm{m}$ feature 
observed in IRAS 06338 is much broader than the $16 \mu \mathrm{m}$ feature we see in HD 52961, and thus probably does not have the same origin. Of course, this $16 \mu \mathrm{m}$ feature could contribute to the observed feature in IRAS 06338, but additional emission is still needed to give rise to such broad emission. Only a few dust species have strong emission features in the $15 \mu \mathrm{m}$ region, most of them are titanium-oxide combination, but none of those species provides an explanation for the observed feature.

A likely explanation for the origin of this broad emission band is that there is a large column of optically thick $\mathrm{CO}_{2}$ gas. Simple isothermal LTE models of optically thick $\mathrm{CO}_{2}$ gas show a broad and strong plateau underneath the strong Q-branch bands. This $\mathrm{CO}_{2}$ plateau is made up by the multitudes of ro-vibrational lines that bunch up in this wavelength range. Such plateaus can indeed reproduce the overall width of the observed emission plateau, but overestimate the flux shortward of $15 \mu \mathrm{m}$. However, if the gas is optically thick, the spectrum can be very sensitive to temperature and density gradients in the gas, and it is thus conceivable that a more adequate model would in fact reproduce the observations quite well. Such is also the case for HR 4049, where optically thick models can simultaneously explain the broad emission plateau and the individual strong Q-branch bands (Malek et al., in prep.).

\section{PAHS}

After the removal of the contribution of the O-rich dust (Sect. 3), we find clear evidence for PAH features in all sample stars. The PAH emission features are generally divided in three classes, depending on the central wavelength of the most prominent features (Peeters et al. 2002). Class A sources have features at 6.22, 7.6 and $8.6 \mu \mathrm{m}$. Class B sources show quite some variability in peak positions, but tend to be shifted more to the red than class $\mathrm{A}$ features. The more rare class $\mathrm{C}$ sources show emission features at $6.3 \mu \mathrm{m}$, no emission near $7.6 \mu \mathrm{m}$, and a broad feature centered around $8.2 \mu \mathrm{m}$, extending beyond $9 \mu \mathrm{m}$.

As already discussed in Sect. 3, the exact identification of the PAH emission in the $8 \mu \mathrm{m}$ region for IRAS 06338 is uncertain. The features at $6.04-6.28-11.28$ and $12.6 \mu \mathrm{m}$ are relatively independent from the underlying dust emission, unlike the $8 \mu \mathrm{m}$ feature, where the peak position can shift from 8 to $8.3 \mu \mathrm{m}$, depending on the composition of the underlying silicate dust (see Fig. 2). When peaking at $8 \mu \mathrm{m}$, the feature resembles more a class B PAH feature. With the peak at $8.3 \mu \mathrm{m}$, the feature is identified as class $\mathrm{C}$.

IRAS 06338 also shows very unusual emission in the $7 \mu \mathrm{m}$ region. Three clear features at $6.63,6.83$ and $7.07 \mu \mathrm{m}$ can be (see inset Fig. 1), together with two smaller features at 6.49 and $7.25 \mu \mathrm{m}$. The features are reminiscent of the narrow features seen in the PAH spectra of Cami et al. (2011b, Fig. 2), where the features are due small PAHs, consisting of less than 30 carbon atoms. Similar features at 6.85 and $7.25 \mu \mathrm{m}$ have been observed in a few other sources and identified with aliphatic hydrocarbons and/or hydrogenated amorphous carbon (Furton et al. 1999; Chiar et al. 2000; Sloan et al. 2007). A small feature at 6.6$6.7 \mu \mathrm{m}$ has been observed in other circumstellar environments, and tentatively identified with PAH emission (Peeters et al. 1999; Werner et al. 2004b; Smith et al. 2007).

The spectrum of IRAS 13258, shortward of $15 \mu \mathrm{m}$, is dominated by PAH emission, with features at $6.02,6.30,8.22$ and $11.30 \mu \mathrm{m}$. The large PAH feature around $8 \mu \mathrm{m}$ is again relatively broad, and appears to be a blend of smaller features, with peaks at $7.65,8.22$ and $8.58 \mu \mathrm{m}$. This broad band resembles the class $\mathrm{C}$ profile but is clearly narrower and has a red wing that resembles

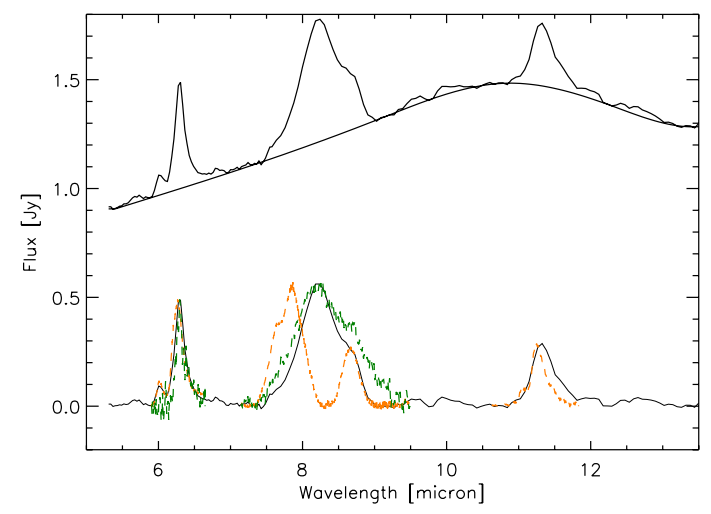

Fig. 6. Identification of the PAH features in IRAS 13258. On the top we plot the spectrum shortwards of $13 \mu \mathrm{m}$, together with a spline fit to represent the underlying continuum. The residual spectrum is given below, together with an example of the class B (orange) and class $\mathrm{C}$ (dark green) PAH features as described by Peeters et al. (2002) and van Diedenhoven et al. (2004).

more a highly redshifted B profile (peaking at $8 \mu \mathrm{m}$ ). Given that the peak position is $8.22 \mu \mathrm{m}$, we classify it as a $\mathrm{C}$ profile. Other small peaks can be seen at 9.1, 9.6, 10.0, 10.6 and $12.6 \mu \mathrm{m}$. The spectrum shows strong similarities with that of EP Lyr (see Fig. 1), which also shows PAHs emission at shorter wavelengths and strong crystalline silicate emission at longer wavelengths (Gielen et al. 2009).

For HD 52961 the residual spectrum (Fig. 3) shows similar $\mathrm{PAH}$ features as for the previous two sources. We again find a broad $8 \mu \mathrm{m}$ feature, with a possible small side feature at $9.2 \mu \mathrm{m}$. This would then make it a BC class (Sloan et al. 2005). The $11.3 \mu \mathrm{m}$ feature is very similar to that observed in IRAS 13258 , with a similar width and central wavelength.

\section{Fullerenes: $\mathbf{C}_{60}$}

As can be seen already from Fig. 1, the spectra of HD 52961 and IRAS 06338 show weak features near 17.4 and $19 \mu \mathrm{m}$ (Fig. 7). These features have recently been identified with the infrared active vibrational modes of neutral $\mathrm{C}_{60}$ in the Spitzer spectrum of the C-rich planetary nebula Tc 1 (Cami et al. 2010). In that source, the bands show fairly broad emission bands with a roughly Gaussian profile, similar as in our objects. However, in our spectra, the short-wavelength bands at 7 and $8.5 \mu \mathrm{m}$ are missing, as are bands of $\mathrm{C}_{70}$.

The two $\mathrm{C}_{60}$ features are weak, and especially the $17.4 \mu \mathrm{m}$ band is furthermore contaminated by some $\mathrm{CO}_{2}$ emission (see Sect. 4). We can get an estimate of the physical conditions and masses involved, following a similar approach as described in Cami et al. (2010), using the emitted power in each of the bands. Given the absence of the bands at shorter wavelengths and contamination with $\mathrm{CO}_{2}$ lines (see Sect. 5), the diagnostic value of the $\mathrm{C}_{60}$ bands is however somewhat limited.

\subsection{HD 52961}

The band profiles of the $\mathrm{C}_{60}$ bands in HD 52961 appear fairly symmetric, and Fig. 7 shows that a gaussian profile reproduces the observations well. From integrating the spectrum, we find that the total power emitted in the $\mathrm{C}_{60}$ bands is $2.6 \pm 0.2 \times 10^{-16}$ and $6.5 \pm 0.2 \times 10^{-16} \mathrm{Wm}^{-2}$ for the 17.4 and $18.9 \mu \mathrm{m}$ bands respectively. From the ratio of these numbers, we then find a nominal excitation temperature of $152_{-22}^{+29} \mathrm{~K}$. At a distance of $2.1 \mathrm{kpc}$, the observed emission requires a total mass of about $3.3 \times 10^{-8} M_{\odot}$. Note that these uncertainties do not include 


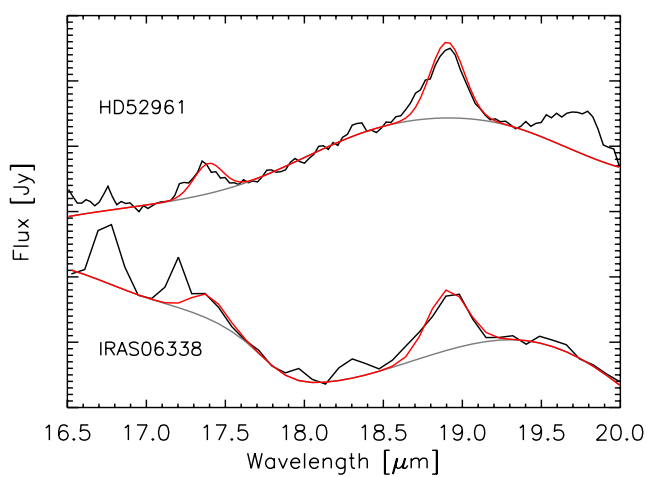

Fig. 7. The observed spectra of HD 52961 and IRAS 06338 (black) with the estimated spline continuum (grey). In red we overplot the Gaussian profiles that were fit to the two $\mathrm{C}_{60}$ bands. The spectra are scaled and offset for comparison.

estimates of systematic errors made by positioning of the continuum level.

The observed widths of the features in HD 52961 are somewhat narrower than in the C-rich planetary nebula Tc 1 (Cami et al. 2010). Smaller widths are expected for lower temperatures, and indeed, also the excitation temperature for HD 52961 is somewhat lower than the $\sim 330 \mathrm{~K}$ found for Tc 1 . Note though that the observed widths are smaller than what would be expected based on laboratory experiments where band widths have been measured at various temperatures (e.g. Nemes et al. 1994). The low temperature also explains the absence of the two bands at shorter wavelengths: at an excitation temperature of $200 \mathrm{~K}$ and assuming thermal excitation, the total power in each of the bands at shorter wavelengths is less than $10 \%$ of the power in the $17.4 \mu \mathrm{m}$ band.

\subsection{IRAS 06338}

The IRAS 06338 observations correspond to a much lower spectral resolution than those of HD 52961, and at the same time the $\mathrm{C}_{60}$ features are weaker, and heavily contaminated by highexcitation emission bands due to $\mathrm{CO}_{2}$. This makes a good determination of the relevant parameters much harder, and any derived quantity is probably rather uncertain given the systematic errors possible on the local continuum. We masked the $\mathrm{CO}_{2}$ bands, and adopted a local continuum, and fitted Gaussian profiles to the bands (See Fig. 7).

The widths (FWHM) of the 17.4 and $18.9 \mu \mathrm{m}$ bands are comparable to what we find for HD 52961. The emitted power is $2.7 \times 10^{-17}$ and $7.0 \times 10^{-17} \mathrm{Wm}^{-2}$ respectively, yielding a nominal excitation temperature of $145 \mathrm{~K}$ and a $\mathrm{C}_{60}$ mass of $1.5 \times 10^{-8} M_{\odot}$ (adopting a distance of $3.9 \mathrm{kpc}$ ).

\section{Discussion}

\subsection{Mineralogy}

In all three sample sources, we find evidence for O-rich silicates dust features. Both IRAS 06338 and HD 52961 have a peculiar silicate mineralogy, very different to what is generally seen in post-AGB disc sources (Gielen et al. 2011). The sources seem to be dominated by warmer dust species, with strong emission in the $10 \mu \mathrm{m}$ region, but very weak features at longer wavelengths. This is also seen in interferometric measurements of HD 52961, where the crystalline grains seem to be concentrated in the inner disc regions (Deroo et al. 2006). The peak position of the $10 \mu \mathrm{m}$ feature in IRAS 06338 points to a lack of amorphous olivine, with a preference for amorphous pyroxene and silica, or alumino-silicates, in stead. However, both at short and long wavelengths we find clear features of forsterite, the crystalline olivine form. HD 52961 shows a $10 \mu \mathrm{m}$ feature which is considerably less broad than other post-AGB disc sources (Gielen et al. 2011), again pointing to the dominance of amorphous pyroxene and silica over amorphous olivine. The star also has unusually strong features at 11.3 and $16 \mu \mathrm{m}$, wavelengths typically associated with forsterite emission. The exact shape and strength of these features proved difficult to model with current synthetic spectra of forsterite.

The underabundance of amorphous olivine and weak crystalline silicate features in IRAS 06338 and HD 52961 show that the dust is rather metal-poor. It remains unclear whether this peculiar mineralogy could be linked to the extreme chemistry in the stellar photosphere. The photosphere for HD 52961 shows a strong deficiency of metals, with $[\mathrm{Fe} / \mathrm{H}]=-4.8$ (Waelkens et al. 1991b), which are currently locked in dust grains in the disc. IRAS 06338, however, does not show such extreme depletion, rather its value of $[\mathrm{Fe} / \mathrm{H}]=-1.6$ and $[\mathrm{Mg} / \mathrm{H}]=-1.5$ (Hrivnak et al. 2008) are average values found for post-AGB disc sources (De Ruyter et al. 2006). Furthermore, HR 4049 has a similar extreme depletion pattern as HD 52961 (Waelkens et al. 1991a), but has a very different infrared spectral signature, with features only due to $\mathrm{CO}_{2}$ gas and class B PAHs, and no silicate emission. Unfortunately, due to this strong depletion pattern, it is very difficult to determine the initial metallicity of the central star.

\section{2. $\mathrm{PAHs}$}

The PAH emission seen in all sample stars can be identified as class $\mathrm{C}$ (or $\mathrm{BC}$ ), depending on the underlying silicate emission (Peeters 2011, and references therein). All our sources follow the trend between the effective temperature of the central star and the central wavelength of the 7-8 $\mu \mathrm{m}$ PAH feature (Sloan et al. 2007; Smolders et al. 2010). The carriers of the class C features are proposed to be aliphatic carbonaceous material (Sloan et al. 2007). If indeed aliphatic carbonaceous material is responsible for the class $\mathrm{C}$ profile, we would expect more aliphatic bands at 6.85 and $7.25 \mu \mathrm{m}$, which are (tentatively) observed in IRAS 06338 and IRAS 13258.

\subsection{Fullerenes}

Even though the diagnostic value of the $\mathrm{C}_{60}$ bands in IRAS 06338 and HD 52961 is rather limited, the derived low excitation temperatures show that the fullerenes cannot be located very close to the central star. Adopting a luminosity of $5000 L_{\odot}$, a dust temperature of $150 \mathrm{~K}$ corresponds to a distance of about 240 AU. Unfortunately, neither for the PAH or fullerene features, we can determine their exact spatial location.

Several possible mechanisms for the formation of fullerenes in the CE of evolved stars are discussed by Cami et al. (2011a). The classic experiments that led to the discovery of fullerenes (Kroto et al. 1985) show that fullerenes self-assemble from collisions between carbon clusters in a H-poor environment. The presence of $\mathrm{H}$ under similar conditions inhibits the formation of fullerenes, and instead results in the formation of PAHs (Kroto \& McKay 1988; de Vries et al. 1993). At much higher temperatures $(>3000 \mathrm{~K})$, fullerenes do form in the presence of hydrogen (Jäger et al. 2009). None of these mechanisms seem to correspond to the conditions that could be expected in the outflows of evolved stars though. The simultaneous presence of fullerenes and PAHs in several PNe led García-Hernández et al. (2010) to conclude that PAHs and fullerenes form from photoprocessing of hydrogenated amorphous carbon (HAC), as was experimentally suggested by e.g. Scott et al. (1997). However, if this 
Table 2. Overview of the different characteristics of post-AGB disc sources with evidence for carbonaceous molecules from the list given by De Ruyter et al. (2006) and Gielen et al. (2011).

\begin{tabular}{lcccrccccc}
\hline \hline Name & Spec. Type & $T_{\text {eff }}(\mathrm{K})$ & $\mathrm{C} / \mathrm{O}$ & $L_{\mathrm{IR}} / L_{*}$ & $\mathrm{Am}$. Sil. & Cryst. Sil. & $\mathrm{PAHs}_{60}$ & $\mathrm{CO}_{2}$ \\
\hline EP Lyr & A4I & 7000 & $<1$ & $3 \%$ & no/weak & strong & class C & no & yes \\
IRAS 06338 & F3I & 6250 & $<1$ & $3 \%$ & yes & weak & class (B)C & yes & yes \\
HD 52961 & F6I & 6000 & $<1$ & $12 \%$ & yes & weak & class C & yes & yes \\
HR 4049 & A6I & 7500 & $<1$ & $25 \%$ & no & no & class B & no & yes \\
IRAS 13258 & F4Ib-G0Ib & $?$ & $?$ & $>50 \%$ & $?$ & strong & class C & no & no \\
Red rectangle & F1I & 7500 & $\leq 1$ & $>100 \%$ & no & strong & class B & no & no \\
\hline
\end{tabular}

Notes. We list the name, spectral type, effective temperature, $\mathrm{C} / \mathrm{O}$ ratio, the $L_{\mathrm{IR}} / L_{*}$ ratio, the presence of amorphous and/or crystalline silicates, the detection of PAHs and/or $\mathrm{C}_{60}$, and the presence of $\mathrm{CO}_{2}$ gas lines in their infrared spectra.

would be the fullerene formation process in PNe, it is hard to understand why fullerenes are only detected in low-excitation $\mathrm{PNe}$, and it would be even more unlikely to invoke such a process for the formation of fullerenes in our post-AGB sources, where the UV irradiation is considerably weaker. One of the more promising avenues is the more recent study of Micelotta et al. (2010), that shows fullerenes could form from the destruction of PAHs due to shocks. Micelotta et al. (2010) find that shocks with velocities between 75 and $100 \mathrm{~km} \mathrm{~s}^{-1}$ are needed to modify PAH molecules in the ISM. For several post-AGB disc sources, shocks due to strong winds (reaching speeds well above $100 \mathrm{~km} \mathrm{~s}^{-1}$ ) and pulsation (due to the crossing of the Cepheid instability strip) have been measured (e.g. Gillet et al. 1989; Van Winckel et al. 1998; Maas et al. 2003; Van Winckel 2003; Maas et al. 2005). Thus, this scenario could well be applicable for the shocked material around these post-AGB binaries, where the higher material densities involved could be beneficiary to the fullerene formation.

\subsection{Carbonaceous molecules in an O-rich environment}

Given the O-rich classification of IRAS 06338, HD 52961 and EPLyr, there must be a process that can free some of the locked carbon in the $\mathrm{CE}$ to form the observed large carbonaceous molecules. $\mathrm{CO}$ molecules can be destroyed due to photodissociation, but the UV radiation of these sources might not be strong enough to dissociate large quantities of CO. Alternatively, $\mathrm{X}$-ray radiation, caused by shocks, is able to break up CO (Woods et al. 2005). Another possibility is the Fischer-Tropsch catalysis mechanism, a chemical reaction process that can form hydrocarbons and $\mathrm{H}_{2} \mathrm{O}$ out of $\mathrm{CO}$ and $\mathrm{H}_{2}$. This mechanism has been invoked to explain the presence of $\mathrm{C}$-rich molecules in other O-rich environments and the solar nebula (Kress \& Tielens 2001; Jura et al. 2006). PAHs have also been found around O-rich PNe central stars (e.g. Guzman-Ramirez et al. 2011), usually surrounded by a dense torus. They present a chemical model that shows that hydrocarbon chains can form within an O-rich environment, but high UV radiation is necessary to break up $\mathrm{CO}$, which is not present in our post-AGB disc sources.

Another scenario could be that the central stars have become C-rich, but that due to the reaccretion of O-rich gas from the circumbinary disc, an optically thick layer is newly formed on the stellar photosphere (Mathis \& Lamers 1992). The effects of this accretion process can be seen in the photospheric depletion of metals, but it is unclear whether the reaccreted gas is rich enough in oxygen, or the accretion rate high enough, to explain the apparent O-rich stellar photosphere. Furthermore, we know that post-AGB stars often undergo strong pulsations (Van Winckel et al. 1999, 2009), which might easily mix the different layers of the stellar photosphere. For IRAS 06338 and HD 52961 pulsation periods of, respectively, 29 and 72 days are found (Waelkens et al. 1991b; Hrivnak et al. 2008).
In the case of IRAS 13258 where the $\mathrm{C} / \mathrm{O}$ ratio is not known, and the central sources could thus be carbon rich, the carbonaceous molecules can be located in a more recent, C-rich outflow, while the silicate dust is located in the O-rich disc.

\subsection{Relation to other characteristics}

The question remains why C-rich molecules are observed in only a few of the larger sample of $\sim 70$ post-AGB disc sources as given in De Ruyter et al. (2006) and Gielen et al. (2011). An overview of the different observational characteristics of these six sources is given in Table 2.

So far, most of the post-AGB disc sources where PAH emission is seen, also show mid-infrared $\mathrm{CO}_{2}$ gas emission; IRAS 13256 and the Red Rectangle being the exception. $\mathrm{No} \mathrm{CO}_{2}$ gas lines are seen in other sources from the larger Spitzer sample presented in Gielen et al. (2011). This could mean that the $\mathrm{CO}_{2}$ gas is linked to the formation of carbonaceous molecules in an O-rich CE; or that an underlying mechanism gives rise to the different observed characteristics. The presence of $\mathrm{CO}_{2}$ gas could be a by-product of the Fischer-Tropsch (FT) catalysis.

Besides the $\mathrm{CO}_{2}$ and PAH emission, IRAS 06338, HD 52961 and EPLyr also all show unusually low $L_{\mathrm{IR}} / L_{*}$ ratios (see Sect. 2). So far, it is unclear whether the low $L_{\mathrm{IR}} / L_{*}$ ratio in these sources is a result of a difference in disc formation or a signature of disc evolution. The low infrared excess could be due to a lower dust mass in these discs, compared to the larger sample. A lower dust-to-gas ratio might then make the gas lines more prominent, explaining the observed strong $\mathrm{CO}_{2}$ emission in these sources. The low infrared excess might point to a disc structure, which is more transparent to stellar photons. This would then increase the photodissociation rate of CO.

\section{Conclusions}

So far, more than 60 infrared spectra of post-AGB disc sources, as listed by De Ruyter et al. (2006), have been studied, both in our own Galaxy and the Large Magellanic Cloud (Waters et al. 1998; Dominik et al. 2003; Gielen et al. 2008, 2011). The spectra are nearly always dominated by emission due to O-rich silicates, with a high degree of crystalline dust. Of this large sample, the total number of sources where we find evidence for species besides silicate dust now comes to six, including the new objects discussed in this paper.

In these six sources the additional features are due to $\mathrm{O}$ rich $\mathrm{CO}_{2}$ gas lines and/or carbonaceous molecules, most often PAHs. Our detection of $\mathrm{C}_{60}$ in HD 52961 and IRAS 06338 represents the first time these molecules are found in binary postAGB stars. The presence of large aromatic molecules in these environments is very surprising and puzzling, given the O-rich nature of the circumbinary disc, and possibly the central star itself. 


\section{Appendix A: Additional table}

Table A.1. Best fit parameters deduced from our full spectral fitting.

\begin{tabular}{|c|c|c|c|c|c|c|c|c|}
\hline & $\begin{array}{c}\text { MgFeOliv } \\
\quad \mathrm{S}-\mathrm{L}\end{array}$ & $\begin{array}{c}\mathrm{MgFePyr} \\
\mathrm{S}-\mathrm{L}\end{array}$ & $\begin{array}{c}\text { MgOliv } \\
\text { S - L }\end{array}$ & $\begin{array}{c}\mathrm{MgPyr} \\
\mathrm{S}-\mathrm{L}\end{array}$ & $\begin{array}{l}\text { Silica } \\
\mathrm{S}-\mathrm{L}\end{array}$ & $\begin{array}{l}\text { Forst } \\
S-L\end{array}$ & $\begin{array}{l}\text { Enst } \\
\text { S - L }\end{array}$ & $\mathrm{NaAlSi}_{4} \mathrm{O}_{10}$ \\
\hline \multicolumn{9}{|l|}{ IRAS 06338} \\
\hline $10 \mu \mathrm{m}$ region $\mathrm{A}$ & $0.0-0.0$ & $68.3-0.0$ & $0.0-0.0$ & $0.0-0.0$ & $0.0-21.3$ & $6.6-0.0$ & $1.4-2.4$ & - \\
\hline $10 \mu \mathrm{m}$ region $\mathrm{B}$ & $0.0-0.0$ & $1.2-0.0$ & $0.0-0.0$ & $0.0-0.0$ & $0.0-0.0$ & $11.6-21.1$ & $3.1-10.9$ & 51.4 \\
\hline $20 \mu \mathrm{m}$ region & - & - & - & - & $0.0-16.5$ & $15.7-41.3$ & $0.0-26.5$ & - \\
\hline \multicolumn{9}{|l|}{ HD 52961} \\
\hline $10 \mu \mathrm{m}$ region & $0.0-0.0$ & $0.0-0.0$ & $32.0-0.0$ & $37.6-0.0$ & $0.0-10.0$ & $20.4-0.0$ & $0.0-0.0$ & - \\
\hline $20 \mu \mathrm{m}$ region & - & - & - & - & $0.0-26.5$ & $17.8-40.2$ & $0.0-15.5$ & - \\
\hline \multicolumn{9}{|l|}{ IRAS 13258} \\
\hline $20 \mu \mathrm{m}$ region & - & - & - & - & $0.0-0.0$ & $22.8-29.6$ & $9.5-38.2$ & - \\
\hline
\end{tabular}

Notes. The abundances of small $(0.1 \mu \mathrm{m})$ and large $(2.0 \mu \mathrm{m})$ grains $(\mathrm{S}-\mathrm{L})$ of the various dust species are given as fractions of the total mass, excluding the dust responsible for the continuum emission.

\section{References}

Bakker, E. J., van Dishoeck, E. F., Waters, L. B. F. M., \& Schoenmaker, T. 1997, A\&A, 323, 469

Beintema, D. A., van den Ancker, M. E., Molster, F. J., et al. 1996, A\&A, 315, L369

Bond, H. E., \& Luck, R. E. 1987, ApJ, 312, 203

Cami, J., Bernard-Salas, J., Peeters, E., \& Malek, S. E. 2010, Science, 329, 1180

Cami, J., Bernard-Salas, J., Peeters, E., \& Malek, S. E. 2011a, in IAU Symp., 280

Cami, J., \& Yamamura, I. 2001, A\&A, 367, L1

Cami, J., Boersma, C., Peeters, E., et al. 2011b, in IAU Symp., 280, 112

Cerrigone, L., Hora, J. L., Umana, G., \& Trigilio, C. 2009, ApJ, 703, 585

Cherchneff, I. 2006, A\&A, 456, 1001

Chiar, J. E., Tielens, A. G. G. M., Whittet, D. C. B., et al. 2000, ApJ, 537, 749

De Ruyter, S., Van Winckel, H., Maas, T., et al. 2006, A\&A, 448, 641

de Vries, M. S., Reihs, K., Wendt, H. R., et al. 1993, Geochim. Cosmochim. Acta, 57, 933

Deroo, P., Van Winckel, H., Min, M., et al. 2006, A\&A, 450, 181

Deroo, P., Van Winckel, H., Verhoelst, T., et al. 2007, A\&A, 467, 1093

Dominik, C., Dullemond, C. P., Cami, J., \& van Winckel, H. 2003, A\&A, 397 595

Dorschner, J., Begemann, B., Henning, T., Jaeger, C., \& Mutschke, H. 1995, A\&A, 300, 503

Duari, D., Cherchneff, I., \& Willacy, K. 1999, A\&A, 341, L47

Furton, D. G., Laiho, J. W., \& Witt, A. N. 1999, ApJ, 526, 752

García-Hernández, D. A., Abia, C., Manchado, A., \& García-Lario, P. 2006, A\&A, 452, 1049

García-Hernández, D. A., Manchado, A., García-Lario, P., et al. 2010, ApJ, 724, L39

García-Hernández, D. A., Iglesias-Groth, S., Acosta-Pulido, J. A., et al. 2011, ApJ, 737, L30

Gielen, C., Van Winckel, H., Waters, L. B. F. M., Min, M., \& Dominik, C. 2007, A\&A, 475, 629

Gielen, C., Van Winckel, H., Min, M., Waters, L. B. F. M., \& Lloyd Evans, T. 2008, A\&A, 490, 725

Gielen, C., van Winckel, H., Matsuura, M., et al. 2009, A\&A, 503, 843

Gielen, C., Bouwman, J., Van Winckel, H., et al. 2011, A\&A, 533, A99

Gillet, D., Duquennoy, A., Bouchet, P., \& Gouiffes, C. 1989, A\&A, 215, 316

Giridhar, S., Lambert, D. L., \& Gonzalez, G. 1998, ApJ, 509, 366

Giridhar, S., Lambert, D. L., \& Gonzalez, G. 2000, ApJ, 531, 521

Giridhar, S., Lambert, D. L., Reddy, B. E., Gonzalez, G., \& Yong, D. 2005, ApJ, 627,432

Gonzalez, G., Lambert, D. L., \& Giridhar, S. 1997, ApJ, 479, 427

Guzman-Ramirez, L., Zijlstra, A. A., Níchuimín, R., et al. 2011, MNRAS, 414, 1667

Henning, T., \& Mutschke, H. 1997, A\&A, 327, 743

Herwig, F. 2005, ARA\&A, 43, 435
Hines et al. 2005, FEPS Data Explanatory Supplement, Version 3.0, Pasadena SSC

Hony, S., Tielens, A. G. G. M., Waters, L. B. F. M., \& de Koter, A. 2003, A\&A, 402,211

Hony, S., Heras, A. M., Molster, F. J., \& Smolders, K. 2009, A\&A, 501, 609

Houck, J. R., Roellig, T. L., van Cleve, J., et al. 2004, ApJS, 154, 18

Hrivnak, B. J., Van Winckel, H., Reyniers, M., et al. 2008, AJ, 136, 1557

Jaeger, C., Molster, F. J., Dorschner, J., et al. 1998, A\&A, 339, 904

Jäger, C., Huisken, F., Mutschke, H., Jansa, I. L., \& Henning, T. 2009, ApJ, 696, 706

Jura, M., Bohac, C. J., Sargent, B., et al. 2006, ApJ, 637, L45

Justtanont, K., de Jong, T., Helmich, F. P., et al. 1996, A\&A, 315, L217

Koike, C., Chihara, H., Tsuchiyama, A., et al. 2003, A\&A, 399, 1101

Koike, C., Imai, Y., Chihara, H., et al. 2010, ApJ, 709, 983

Kress, M. E., \& Tielens, A. G. G. M. 2001, Meteoritics and Planetary Science, 36,75

Kroto, H. W., \& McKay, K. 1988, Nature, 331, 328

Kroto, H. W., Heath, J. R., Obrien, S. C., Curl, R. F., \& Smalley, R. E. 1985, Nature, 318,162

Lahuis et al. 2006, c2d Spectroscopy Explanatory Supplement, Cores to Disks, SpitzeR Legacy Team, (Pasadena: Spitzer Science Center)

Lloyd Evans, T. 1997, Ap\&SS, 251, 239

Luck, R. E., \& Bond, H. E. 1984, ApJ, 279, 729

Maas, T., Van Winckel, H., \& Lloyd Evans, T. 2003, in ASP Conf. Ser., 303, ed. R. L. M. Corradi, J. Mikolajewska, \& T. J. Mahoney, 143

Maas, T., Van Winckel, H., \& Lloyd Evans, T. 2005, A\&A, 429, 297

Mathis, J. S., \& Lamers, H. J. G. L. M. 1992, A\&A, 259, L39

Matsuura, M., Zijlstra, A. A., Molster, F. J., et al. 2004, ApJ, 604, 791

Matsuura, M., Wood, P. R., Sloan, G. C., et al. 2006, MNRAS, 371, 415

Micelotta, E. R., Jones, A. P., \& Tielens, A. G. G. M. 2010, A\&A, 510, A36

Molster, F. J., Yamamura, I., Waters, L. B. F., et al. 2001, A\&A, 366, 923

Molster, F. J., Waters, L. B. F. M., \& Tielens, A. G. G. M. 2002a, A\&A, 382, 222

Molster, F. J., Waters, L. B. F. M., Tielens, A. G. G. M., \& Barlow, M. J. 2002b, A\&A, 382, 184

Mutschke, H., Begemann, B., Dorschner, J., et al. 1998, A\&A, 333, 188

Nejad, L. A. M., \& Millar, T. J. 1988, MNRAS, 230, 79

Nemes, L., Ram, R. S., Bernath, P. F., et al. 1994, Chem. Phys. Lett., 218, 295

Peeters, E. 2011, in EAS Publ. Ser., 46, 13

Peeters, E., Tielens, A. G. G. M., Roelfsema, P. R., \& Cox, P. 1999, in The Universe as Seen by ISO, ed. P. Cox, \& M. Kessler, ESA Special Publ., 427, 739

Peeters, E., Hony, S., Van Kerckhoven, C., et al. 2002, A\&A, 390, 1089

Reyniers, M., \& Van Winckel, H. 2007, A\&A, 463, L1

Sargent, B. A., Forrest, W. J., Tayrien, C., et al. 2009, ApJ, 690, 1193

Scott, A., Duley, W. W., \& Pinho, G. P. 1997, ApJ, 489, L193

Servoin, J. L., \& Pirou, B. 1973, Phys. Stat. Sol. B, 55, 677

Shkuratov, Y. G., \& Grynko, Y. S. 2005, Icarus, 173, 16 
C. Gielen et al.: Carbonaceous molecules in the O-rich CE of binary post-AGB stars

Sloan, G. C., Keller, L. D., Forrest, W. J., et al. 2005, ApJ, 632, 956 Sloan, G. C., Jura, M., Duley, W. W., et al. 2007, ApJ, 664, 1144 Smith, J. D. T., Draine, B. T., Dale, D. A., et al. 2007, ApJ, 656, 770 Smolders, K., Acke, B., Verhoelst, T., et al. 2010, A\&A, 514, L1

Speck, A. K., Barlow, M. J., \& Skinner, C. J. 1997, MNRAS, 288, 431

Szczerba, R., Schmidt, M. R., \& Pulecka, M. 2007, Baltic Astron., 16, 134

van Diedenhoven, B., Peeters, E., Van Kerckhoven, C., et al. 2004, ApJ, 611, 928 Van Winckel, H. 2003, ARA\&A, 41, 391

Van Winckel, H., Mathis, J. S., \& Waelkens, C. 1992, Nature, 356, 500

Van Winckel, H., Waelkens, C., Waters, L. B. F. M., et al. 1998, A\&A, 336, L17 Van Winckel, H., Waelkens, C., Fernie, J. D., \& Waters, L. B. F. M. 1999, A\&A, 343, 202

Van Winckel, H., Lloyd Evans, T., Briquet, M., et al. 2009, A\&A, 505, 1221

Volk, K., Hrivnak, B. J., Matsuura, M., et al. 2011, ApJ, 735, 127
Waelkens, C., Lamers, H. J. G. L. M., Waters, L. B. F. M., et al. 1991a, A\&A 242,433

Waelkens, C., Van Winckel, H., Bogaert, E., \& Trams, N. R. 1991b, A\&A, 251, 495

Waters, L. B. F. M., Trams, N. R., \& Waelkens, C. 1992, A\&A, 262, L37

Waters, L. B. F. M., Molster, F. J., de Jong, T., et al. 1996, A\&A, 315, L361

Waters, L. B. F. M., Cami, J., de Jong, T., et al. 1998, Nature, 391, 868

Werner, M. W., Roellig, T. L., Low, F. J., et al. 2004a, ApJS, 154, 1

Werner, M. W., Uchida, K. I., Sellgren, K., et al. 2004b, ApJS, 154, 309

Woods, P. M., Nyman, L.-Å, Schöier, F. L., et al. 2005, A\&A, 429, 977

Yamamura, I., Dominik, C., de Jong, T., Waters, L. B. F. M., \& Molster, F. J. 2000, A\&A, 363, 629

Zhang, Y., \& Kwok, S. 2011, ApJ, 730, 126 